\title{
Recombination line intensities for hydrogenic ions: The fine-structure components of $\mathrm{H} I$ and $\mathrm{He} I I^{\star}$
}

\author{
R.E.S. Clegg ${ }^{1}$, S. Miller ${ }^{2}$, P.J. Storey ${ }^{2}$, and R. Kisielius ${ }^{2}$ \\ 1 Particle Physics and Astronomy Research Council, Polaris House, North Star Avenue, Swindon, Wiltshire SN2 1SZ, UK \\ 2 Department of Physics and Astronomy, University College London, Gower Street, London WC1E 6BT, UK
}

Received July 1, 1993; accepted October 21, 1998

\begin{abstract}
Emissivities have been calculated for finestructure components of selected UV and optical recombination lines of $\mathrm{HI}$ and HeII. Results are given for a range of electron temperatures and densities, and in Cases A and B of Baker \& Menzel (1938). Relative intensities, wavelengths and velocity shifts of the fine-structure components are tabulated. Applications to the spectra of gaseous nebulae are discussed. Depending on the temperature, density and Case, the centres of unresolved lines can shift by up to $0.9 \mathrm{~km} \mathrm{~s}^{-1}$ for $\mathrm{H}$ I and $3.3 \mathrm{~km} \mathrm{~s}^{-1}$ for He II. A new calibration of the $\mathrm{H} \alpha-[\mathrm{N} \mathrm{II}]$ method for determining electron temperatures is given.
\end{abstract}

Key words: atomic data - line: formation - line: profiles - H II regions — planetary nebulae: general

\section{Introduction}

Recombination lines of hydrogen and helium in the optical, IR and UV spectral regions are frequently used in astronomical spectroscopy for measurements of radial velocities and velocity fields. The levels of hydrogenic ions are not quite degenerate, and lines such as $\mathrm{H} \alpha(n=3 \rightarrow 2)$ and He II $4686 \AA(n=4 \rightarrow 3)$ consists of many components; for example for $\mathrm{H} \alpha$ there are seven components, with transitions between the $3 \mathrm{~d}^{2} \mathrm{D}_{5 / 2,3 / 2}, 3 \mathrm{p}^{2} \mathrm{P}_{3 / 2,1 / 2}$ and $3 \mathrm{~s}^{2} \mathrm{~S}_{1 / 2}$ in $n=3$ and $2 \mathrm{p}^{2} \mathrm{P}_{3 / 2,1 / 2}$ and $2 \mathrm{~s}^{2} \mathrm{~S}_{1 / 2}$ in $n=2$, subject to the usual selection rules for dipole allowed transitions. He II $\lambda 4686 \AA$ has thirteen components.

The precise energies of these levels in hydrogenic ions depend on relativistic shifts as well as fine-structure effects

Send offprint requests to: P.J. Storey

* All tables are also available in electronic form at the CDS via anonymous ftp to cdsarc.u-strasbg.fr (130.79.128.5) or via http://cdsweb.u-strasbg.fr/Abstract.html derived from the spin-orbit interaction and quantum electrodynamic effects such as the Lamb shift. For example, the $2 \mathrm{~s}^{2} \mathrm{~S}_{1 / 2}$ and $2 \mathrm{p}^{2} \mathrm{P}_{1 / 2}$ states of $\mathrm{HI}$ are separated by $0.0353 \mathrm{~cm}^{-1}$ due to QED effects, while the $2 \mathrm{p}^{2} \mathrm{P}_{1 / 2}$ and $2 \mathrm{p}^{2} \mathrm{P}_{3 / 2}$ states are separated by $0.3659 \mathrm{~cm}^{-1}$ due to the spin-orbit interaction.

Unless the line components are resolved, their effect is to broaden the spectral lines and to produce small shifts in the effective position of the line centre, depending on the distribution of intensities between the components. As we show below, the range covered by the line components is $9.1 \mathrm{~km} \mathrm{~s}^{-1}$ for hydrogen and $36.6 \mathrm{~km} \mathrm{~s}^{-1}$ for helium lines. In an atom of mass $m$ at temperature $T_{\mathrm{e}}$, the full line width $(F W H M)$ of a single component in velocity units due to thermal broadening is $\left(8 k T_{\mathrm{e}} \ln 2 / m\right)^{1 / 2}$. For $\mathrm{HI}$ and HeII, this width has the values of 21.4 and $10.7 \mathrm{~km} \mathrm{~s}^{-1}$ at $10^{4} \mathrm{~K}$, and 6.8 and $3.4 \mathrm{~km} \mathrm{~s}^{-1}$ at $10^{3} \mathrm{~K}$, respectively. Hence the fine-structure broadening can affect line widths of hydrogen and especially helium recombination lines in cool $\left(T_{\mathrm{e}} \sim 10^{3} \mathrm{~K}\right)$ plasmas. Although many planetary nebulae are somewhat warmer, with $T_{\mathrm{e}} \sim 10^{4} \mathrm{~K}$, H II regions often have $T_{\mathrm{e}} \sim 5000 \mathrm{~K}$ and some old novae have electron temperatures as low as $500 \mathrm{~K}$ (e.g., DQ Her, Williams et al. 1978).

Because of the combined effects of thermal, turbulent and expansion broadening, we do not expect these components to be completely resolved in observations of ionized plasmas in space. However, they are resolved in laboratory experiments using laser techniques (e.g., Hänsch et al. 1975).

We give several examples of the significance of these splittings. Dyson \& Meaburn (1971) showed that the finestructure of the $\mathrm{H} \alpha$ line profile makes a significant difference to derived electron temperatures in nebulae when these are obtained from the ratio of observed widths of the $[\mathrm{N}$ II $] 6584 \AA$ and $\mathrm{H} \alpha$ lines. In a typical example, the temperature derived for the Orion region changed from 6200 to $7350 \mathrm{~K}$, which would alter the derived $\mathrm{O} / \mathrm{H}$ ratio 
(from $[\mathrm{O}$ II $] 3727 \AA$ ) by a factor 2.6. In the present paper we update the $\mathrm{H} \alpha-[\mathrm{N}$ II $]$ correction scheme.

A second application is provided by supernova 1987A. Cumming \& Meikle (1993) detected a remarkable shortlived narrow component in $\mathrm{H} \alpha$ and $\mathrm{H} \beta$ emission from the circumstellar medium of the supernova. In some spatial positions the narrow component's $F W H M$ was as low as $5.3 \pm 1.3 \mathrm{~km} \mathrm{~s}^{-1}$, which after allowance for the $\mathrm{H} \alpha$ finestructure suggests emission from extremely cool recombining gas. In fact, the formal best fit at some positions was narrower than the spread of the fine-structure components. Of several models discussed, one involved illumination of a circumstellar $\mathrm{H}$ I cloud by the $\mathrm{H}$ Ly $\beta$ emission line (absorption of $\mathrm{Ly} \beta$ would only populate the $3 \mathrm{p}$ level, and thus the number of components making up the $\mathrm{H} \alpha$ line would be reduced).

Other studies have revealed narrow emission lines from ionized regions. Onello \& Phillips (1993) observed emission components with $F W H M$ as low as $3.6 \mathrm{kms}^{-1}$ in the $\mathrm{H} 168 \alpha$ recombination line towards galactic sources; such components if seen in the Balmer lines would be affected by the fine-structure we discuss here. Gallagher \& Hunter (1983) studied widths of the $\mathrm{H} \alpha$ line in extragalactic H II regions. Observed widths were corrected for instrumental and thermal broadening, and the "excess widths" due to the bulk gas velocity dispersion were as small as $1.6-13.0 \mathrm{~km} \mathrm{~s}^{-1}(F W H M)$ for some positions in NGC 4214. Correction for fine-structure broadening would reduce these excesses still further.

Lastly, we show below that the He II line at $1640 \AA$ has seven components spanning a range of $36.6 \mathrm{~km} \mathrm{~s}^{-1}$ (with the two strongest components $26 \mathrm{~km} \mathrm{~s}^{-1}$ apart). Laming \& Feldman (1993) analysed SKYLAB spectra of a Solar Prominence which partially resolved the 7 components, and were able to derive information on the process populating the He II $n=3$ levels in the hot plasma.

Calculations of the recombination spectra of hydrogenic ions in Case B of Baker \& Menzel (1938) have been described by Hummer \& Storey (1987), Storey \& Hummer (1988) and Storey \& Hummer (1995) (hereafter HS, SH and SH95). Their calculations take full account of all radiative and collisional processes, and they tabulated emissivities at a range of electron temperatures and densities for principal quantum numbers up to $n=50$.

Martin (1988) presented effective recombination coefficients for $\mathrm{HI}$ and He II states as a function of $n$ and $\ell$, from which the relative intensities of fine-structure components can be derived, but only for the "zero density" case, in which all collisional processes are ignored. Effective recombination coefficients for hydrogen have also been calculated by Smits (1991) as a function of both electron temperature and density. His calculations extend the calculations of Hummer \& Storey (1987) to low electron temperatures, $312.5 \mathrm{~K} \leq T_{\mathrm{e}} \leq 2500 \mathrm{~K}$, using essentially the same methods. He does not, however, treat HeII, or give the $\ell$-dependent information necessary to calculate the intensities of the fine-structure components.

In this paper we summarize the effects of the finestructure components and present new calculations of their relative intensities, as a function of electron temperature and density, and in Cases $\mathrm{A}$ and $\mathrm{B}$. We also include synthetic spectra showing the components in velocity space, and tabulate the shifts of the line centres for different densities, temperatures and Cases.

\section{Adopted energy levels and "reference" wavelengths}

For H I and He II energy levels we adopt the values given by Erickson (1977). These are based on quantum electrodynamic calculations including the Lamb shift. This work makes a careful comparison between experimental and calculated energy-level differences, and demonstrates good agreement. In general the theoretical QED uncertainty is smaller than the experimental error.

For each transition of $\mathrm{HI}$ and He II considered, we adopted a reference wavelength, $\lambda_{\text {ref }}$, for use as a zeropoint in the presentation of the shifts of individual component lines in velocity space. These were computed from the Rydberg formula

$\lambda_{\text {ref }}=\left[\mu R_{\infty}\left(n_{\mathrm{l}}^{-2}-n_{\mathrm{u}}^{-2}\right)\right]^{-1}$,

where $R_{\infty}=109737.3153 \mathrm{~cm}^{-1}$ is the Rydberg constant for infinite nuclear mass (Ferguson 1986), and $\mu$ is the reduced mass of the electron-nucleus system in electron masses. The finite mass Rydberg constants, $\mu R_{\infty}$, that were used to calculate the reference wavelengths are 109677.6155, 109707.4343, 109717.3590 and $109722.2772 \mathrm{~cm}^{-1}$ for ${ }^{1} \mathrm{H}$, ${ }^{2} \mathrm{D},{ }^{3} \mathrm{He}$ and ${ }^{4} \mathrm{He}$ respectively.

The difference in energy, $\Delta_{n j}$, between the energy of a hydrogenic state $E_{n}$, given by the Rydberg formula and the energy $E_{n j}$ obtained from the Dirac equation is approximately

$\Delta_{n j}=E_{n j}-E_{n}=-\frac{Z^{4} \alpha^{4}}{n^{4}} \mu R_{\infty}\left(\frac{n}{j+\frac{1}{2}}-\frac{3}{4}\right)$,

where $\alpha$ is the fine-structure constant and terms in higher powers of $(Z \alpha)$ have been neglected. This equation describes the relativistic energy shifts of the hydrogenic levels (the mass correction and the Darwin term) as well as the fine-structure due to the spin-orbit interaction. The Lamb shift is not included but can be neglected for the argument made here. For a given $n,\left(j+\frac{1}{2}\right)$ runs from one to $n$, so that the bracket in Eq. (2) is always positive. Thus the levels $n \ell j$ always lie energetically lower than the corresponding non-relativistic energies given from the Rydberg formula. In addition, the energy shift $\Delta_{n j}$ falls rapidly as $n$ increases. As a consequence, the fine-structure components in any transition $n_{\mathrm{u}} \rightarrow n_{1}$ will generally be displaced to wavelengths shorter than $\lambda_{0}$ as given by Eq. (1), and the corresponding velocity shifts will usually be negative. 
We ignore hyperfine structure in this work. Its effect is to double the energy levels of ${ }^{1} \mathrm{H}$ and ${ }^{3} \mathrm{He}$, which have finite nuclear spin. The typical splitting is only $0.001 \mathrm{~cm}^{-1}$, which corresponds to a velocity splitting of $0.02 \mathrm{~km} \mathrm{~s}^{-1}$ for optical and UV lines, and is thus neglected.

Table 1 lists, for some commonly observed transitions of $\mathrm{H}$ and $\mathrm{He}$, the number of components $N_{\mathrm{c}}$, the total range in velocity space of the components $\Delta v$, and the reference wavelength, defined as above, for the four isotopes ${ }^{1} \mathrm{H},{ }^{2} \mathrm{D},{ }^{3} \mathrm{He}$ and ${ }^{4} \mathrm{He}$. We stress that the reference wavelength does not represent any mean wavelength for the line in question, but is simply a reference point to anchor our chosen scale of velocity shifts. Here, and throughout this paper, wavelengths are given in air for $\lambda>2000 \AA$ and in vacuum otherwise. The variation of the velocity range of the components as a function of nuclear mass (e.g. between ${ }^{1} \mathrm{H}$ and ${ }^{2} \mathrm{H}$, or ${ }^{3} \mathrm{He}$ and ${ }^{4} \mathrm{He}$ ) is too small to be recorded in Table 1.

\section{Calculation of level populations and emissivities}

\subsection{Calculation of $b_{n \ell}$}

As in earlier calculations (HS and $\mathrm{SH}$ ), we work in terms of the departure coefficients, $b_{n \ell}$, defined in terms of the Saha-Boltzmann populations at electron temperature $T_{\mathrm{e}}$ by

$$
\frac{N_{n \ell}}{N_{\mathrm{e}} N_{+}}=\frac{\omega_{n \ell}}{2 \omega_{+}}\left(\frac{h^{2}}{2 \pi m k T_{\mathrm{e}}}\right)^{3 / 2} \mathrm{e}^{x_{n \ell}} b_{n \ell},
$$

where $N_{\mathrm{e}}$ and $N_{+}$are the number densities of electrons and recombining ions respectively, $x_{n \ell}=E_{n \ell} / k T_{\mathrm{e}}$, and $E_{n \ell}$ is the ionization energy of the state with quantum numbers $n, \ell$. The statistical weights of this state and of the ion ground state are $\omega_{n \ell}$ and $\omega_{+}$. A two stage process is used to compute the values of $b_{n \ell}$.

In the first stage, it is assumed that for a given $n$ the states $n \ell$ are populated in proportion to their statistical weight, so that $b_{n \ell}=b_{n}$ for all $\ell$. This calculation is complete in the sense that a matrix condensation method is used to reduce the infinite set of algebraic equations determining the $b_{n}$ to a finite set. All spontaneous radiative processes and electron induced collisional processes are included. The approximations used for these rates were given in $\mathrm{HS}$.

In the second stage, the equations that determine $b_{n \ell}$ are solved by an iterative procedure. Above some limiting principal quantum number $\left(n=n_{\mathrm{c}}\right)$, we assume that $b_{n \ell}=b_{n}$ and the values calculated in the first stage are used. For $n \leq n_{\mathrm{c}}$, the values of $b_{n \ell}$ are determined explicitly including the effects of $\ell$-changing collisions. The effect of collisions with electrons, protons and ionized helium atoms are included. For the calculation of the hydrogen spectrum, we assume that helium is singly ionized, whereas for the He II spectrum, we assume helium is doubly ionized. The helium abundance is taken to be $10 \%$ of that of hydrogen. An iterative solution for the $b_{n \ell}$ is carried out, starting at $n=n_{\mathrm{c}}$ and ending at $n=2$ with a maximum of twenty iterations being carried out. Above $n=50$, the values of $b_{n \ell}$ are interpolated as a function of $n$. This interpolation scheme and the approximations used for the required $\ell$-dependent rate coefficients are described in HS.

\subsection{Calculation of emissivities for fine-structure components}

The calculation of $b_{n \ell}$ described in Sect. 2.1 allows us to determine the emissivity in the hydrogenic transition $n_{\mathrm{u}} \ell_{\mathrm{u}} \rightarrow n_{\mathrm{l}} \ell_{\mathrm{l}}$

$\varepsilon\left(n_{\mathrm{u}} \ell_{\mathrm{u}} \rightarrow n_{\mathrm{l}} \ell_{\mathrm{l}}\right)=N\left(n_{\mathrm{u}} \ell_{\mathrm{u}}\right) A\left(n_{\mathrm{u}} \ell_{\mathrm{u}} \rightarrow n_{\mathrm{l}} \ell_{\mathrm{l}}\right) h \nu_{\mathrm{ul}}$,

where $A$ is a radiative transition probability and $h \nu_{\mathrm{ul}}$ is the transition energy. The emissivity of a fine-structure component of this transition is

$$
\begin{aligned}
\varepsilon\left(n_{\mathrm{u}} \ell_{\mathrm{u}} j_{\mathrm{u}} \rightarrow n_{\mathrm{l}} \ell_{\mathrm{l}} j_{\mathrm{l}}\right) & =N\left(n_{\mathrm{u}} \ell_{\mathrm{u}} j_{\mathrm{u}}\right) \times \\
& \times A\left(n_{\mathrm{u}} \ell_{\mathrm{u}} j_{\mathrm{u}} \rightarrow n_{\mathrm{l}} \ell_{\mathrm{l}} j_{\mathrm{l}}\right) h \nu_{\mathrm{ul}} .
\end{aligned}
$$

In $L S$-coupling, the transition probabilities in Eqs. (4) and (5) are related by

$$
\begin{aligned}
A\left(n_{\mathrm{u}} \ell_{\mathrm{u}} j_{\mathrm{u}} \rightarrow n_{\mathrm{l}} \ell_{\mathrm{l}} j_{\mathrm{l}}\right) & =\left(2 j_{\mathrm{l}}+1\right)\left(2 \ell_{\mathrm{u}}+1\right) \times \\
& \times\left\{\begin{array}{ccc}
j_{\mathrm{u}} & j_{\mathrm{l}} & 1 \\
\ell_{\mathrm{l}} & \ell_{\mathrm{u}} & \frac{1}{2}
\end{array}\right\}^{2} A\left(n_{\mathrm{u}} \ell_{\mathrm{u}} \rightarrow n_{\mathrm{l}} \ell_{\mathrm{l}}\right),
\end{aligned}
$$

where \{\} is a $6-j$ symbol as defined for example by Brink \& Satchler (1968). In this expression we have assumed that the fine-structure energy shifts are negligible compared to the transition energies. We further assume that $b_{n \ell j}=b_{n \ell}$, with the values of $b_{n \ell}$ being calculated as described above. This is equivalent to the assumption that the $j$-levels associated with a particular $n \ell$ are populated according to their statistical weights $(2 j+1)$.

Techniques for the rapid calculation of the values of $A\left(n_{\mathrm{u}} \ell_{\mathrm{u}} \rightarrow n_{\mathrm{l}} \ell_{\mathrm{l}}\right)$ have been described by Storey \& Hummer (1991).

\section{Results}

We have computed recombination coefficients in Case A and Case $\mathrm{B}$ for $\mathrm{H} \alpha(n=3 \rightarrow 2), \mathrm{H} \beta(n=4 \rightarrow 2)$, $\mathrm{H} \gamma(n=5 \rightarrow 2)$; and He II $4686 \AA(n=4 \rightarrow 3), 3203 \AA$ $(n=5 \rightarrow 3), 1640 \AA(n=3 \rightarrow 2)$ and $1215 \AA(n=4 \rightarrow 2)$. These were selected either as strong optical lines that are commonly used for velocity measurements, or as strong ultraviolet lines which can be resolved by the Hubble Space Telescope. For hydrogen, the selected ranges of electron density and temperature are $10^{2}-10^{9} \mathrm{~cm}^{-3}$ and $300-30000 \mathrm{~K}$. For He II these ranges are $10^{4}-10^{9} \mathrm{~cm}^{-3}$, and $1000-30000 \mathrm{~K}$. The temperature range spans that seen in photo-ionized nebulae, from novae $\left(T_{\mathrm{e}}\right.$ down to $300 \mathrm{~K})$ to the hottest planetary nebulae $\left(T_{\mathrm{e}} \sim 25000 \mathrm{~K}\right)$. 
Table 1. Summary of components, and reference wavelengths of hydrogenic transitions

\begin{tabular}{|c|c|c|c|c|c|c|c|c|}
\hline \multirow[b]{2}{*}{$n_{\mathrm{u}}$} & \multirow[b]{2}{*}{$n_{1}$} & \multirow[b]{2}{*}{$N_{\mathrm{c}}$} & \multicolumn{2}{|c|}{$\Delta v\left(\mathrm{~km} \mathrm{~s}^{-1}\right)$} & \multicolumn{4}{|c|}{ Reference wavelengths $\lambda_{\text {ref }}(\AA)$} \\
\hline & & & $\mathrm{HI}$ & He II & ${ }^{1} \mathrm{HI}$ & ${ }^{2} \mathrm{HI}$ & ${ }^{3} \mathrm{He}$ II & ${ }^{4} \mathrm{He}$ II \\
\hline 2 & 1 & 2 & 1.33 & 5.33 & 1215.6841 & 1215.3537 & 303.8109 & 303.7973 \\
\hline 3 & 1 & 2 & 0.33 & 1.33 & 1025.7335 & 1025.4547 & 256.3405 & 256.3290 \\
\hline 4 & 1 & 2 & 0.13 & 0.53 & 972.5473 & 972.2829 & 243.0487 & 243.0379 \\
\hline 5 & 1 & 2 & 0.07 & 0.27 & 949.7532 & 949.4951 & 237.3523 & 237.3417 \\
\hline 6 & 1 & 2 & 0.04 & 0.15 & 937.8134 & 937.5585 & 234.3684 & 234.3579 \\
\hline 3 & 2 & 7 & 9.12 & 36.64 & 6562.8812 & 6561.0974 & 1640.5790 & 1640.5055 \\
\hline 4 & 2 & 7 & 5.93 & 23.78 & 4861.3784 & 4860.0571 & 1215.2437 & 1215.1893 \\
\hline 5 & 2 & 7 & 5.04 & 20.17 & 4340.5086 & 4339.3288 & 1085.0391 & 1084.9904 \\
\hline 6 & 2 & 7 & 4.65 & 18.61 & 4101.7762 & 4100.6613 & 1025.3619 & 1025.3159 \\
\hline 4 & 3 & 13 & 8.65 & 34.62 & 18751.1492 & 18746.0526 & 4686.0572 & 4685.8471 \\
\hline 5 & 3 & 13 & 5.54 & 22.19 & 12818.1620 & 12814.6780 & 3203.3302 & 3203.1867 \\
\hline 6 & 3 & 13 & 4.73 & 18.94 & 10938.1610 & 10935.1880 & 2733.4893 & 2733.3668 \\
\hline 5 & 4 & 19 & 8.39 & 33.55 & 40511.7561 & 40500.7449 & 10124.2560 & 10123.8022 \\
\hline 6 & 4 & 19 & 5.04 & 20.15 & 26251.6145 & 26244.4793 & 6560.5039 & 6560.2098 \\
\hline 6 & 5 & 25 & 8.27 & 33.02 & 74578.4650 & 74558.1943 & 18637.8548 & 18637.0193 \\
\hline
\end{tabular}

Table 2a. Fine structure components for $n \rightarrow 2$ transitions

\begin{tabular}{rlllllll}
\hline Index & \multicolumn{2}{c}{ Transition } & $\ell_{\mathrm{u}}$ & $2 j_{\mathrm{u}}$ & $\ell_{1}$ & $2 j_{1}$ \\
\hline 1 & $n \mathrm{~s}{ }^{2} \mathrm{~S}_{1 / 2}$ & - & $2 \mathrm{p}{ }^{2} \mathrm{P}_{1 / 2}^{\mathrm{o}}$ & 0 & 1 & 1 & 1 \\
2 & $n \mathrm{~s}{ }^{2} \mathrm{~S}_{1 / 2}$ & - & $2 \mathrm{p}{ }^{2} \mathrm{P}_{3 / 2}^{\mathrm{o}}$ & 0 & 1 & 1 & 3 \\
3 & $n \mathrm{p}{ }^{2} \mathrm{P}_{1 / 2}^{\mathrm{o}}$ & - & $2 \mathrm{~s}{ }^{2} \mathrm{~S}_{1 / 2}$ & 1 & 1 & 0 & 1 \\
4 & $n \mathrm{p}{ }^{2} \mathrm{P}_{3 / 2}^{\mathrm{o}}$ & - & $2 \mathrm{~s}{ }^{2} \mathrm{~S}_{1 / 2}$ & 1 & 3 & 0 & 1 \\
5 & $n \mathrm{~d}{ }^{2} \mathrm{D}_{3 / 2}$ & - & $2 \mathrm{p}{ }^{2} \mathrm{P}_{1 / 2}^{\mathrm{o}}$ & 2 & 3 & 1 & 1 \\
6 & $n \mathrm{~d}{ }^{2} \mathrm{D}_{3 / 2}$ & - & $2 \mathrm{p}{ }^{2} \mathrm{P}_{3 / 2}^{\mathrm{o}}$ & 2 & 3 & 1 & 3 \\
7 & $n \mathrm{~d}{ }^{2} \mathrm{D}_{5 / 2}$ & - & $2 \mathrm{p}{ }^{2} \mathrm{P}_{3 / 2}^{\mathrm{o}}$ & 2 & 5 & 1 & 3 \\
\hline
\end{tabular}

Table 2b. Fine structure components for $n \rightarrow 3$ transitions

\begin{tabular}{rccccccc}
\hline Index & \multicolumn{2}{c}{ Transition } & $\ell_{\mathrm{u}}$ & $2 j_{\mathrm{u}}$ & $\ell_{\mathrm{l}}$ & $2 j_{1}$ \\
\hline 1 & $n \mathrm{~s}{ }^{2} \mathrm{~S}_{1 / 2}$ & - & $3 \mathrm{p}{ }^{2} \mathrm{P}_{1 / 2}^{\mathrm{o}}$ & 0 & 1 & 1 & 1 \\
2 & $n \mathrm{~s}{ }^{2} \mathrm{~S}_{1 / 2}$ & - & $3 \mathrm{p}{ }^{2} \mathrm{P}_{3 / 2}^{\mathrm{o}}$ & 0 & 1 & 1 & 3 \\
3 & $n \mathrm{p}{ }^{2} \mathrm{P}_{1 / 2}^{\mathrm{o}}$ & - & $3 \mathrm{~s}{ }^{2} \mathrm{~S}_{1 / 2}$ & 1 & 1 & 0 & 1 \\
4 & $n \mathrm{p}{ }^{2} \mathrm{P}_{3 / 2}^{\mathrm{o}}$ & - & $3 \mathrm{~s}{ }^{2} \mathrm{~S}_{1 / 2}$ & 1 & 3 & 0 & 1 \\
5 & $n \mathrm{p}{ }^{2} \mathrm{P}_{1 / 2}^{\mathrm{o}}$ & - & $3 \mathrm{~d}{ }^{2} \mathrm{D}_{3 / 2}$ & 1 & 1 & 2 & 3 \\
6 & $n \mathrm{p}{ }^{2} \mathrm{P}_{3 / 2}^{\mathrm{o}}$ & - & $3 \mathrm{~d}{ }^{2} \mathrm{D}_{3 / 2}$ & 1 & 3 & 2 & 3 \\
7 & $n \mathrm{p}{ }^{2} \mathrm{P}_{3 / 2}^{\mathrm{o}}$ & - & $3 \mathrm{~d}{ }^{2} \mathrm{D}_{5 / 2}$ & 1 & 3 & 2 & 5 \\
8 & $n \mathrm{~d}{ }^{2} \mathrm{D}_{3 / 2}$ & - & $3 \mathrm{p}^{2} \mathrm{P}_{1 / 2}^{\mathrm{o}}$ & 2 & 3 & 1 & 1 \\
9 & $n \mathrm{~d}{ }^{2} \mathrm{D}_{3 / 2}$ & - & $3 \mathrm{p}{ }^{2} \mathrm{P}_{3 / 2}^{\mathrm{o}}$ & 2 & 3 & 1 & 3 \\
10 & $n \mathrm{~d}{ }^{2} \mathrm{D}_{5 / 2}$ & - & $3 \mathrm{p}{ }^{2} \mathrm{P}_{3 / 2}^{\mathrm{o}}$ & 2 & 5 & 1 & 3 \\
11 & $n \mathrm{f}{ }^{2} \mathrm{~F}_{5 / 2}^{\mathrm{o}}$ & - & $3 \mathrm{~d}^{2} \mathrm{D}_{3 / 2}$ & 3 & 5 & 2 & 3 \\
12 & $n \mathrm{f}{ }^{2} \mathrm{~F}_{5 / 2}^{\mathrm{o}}$ & - & $3 \mathrm{~d}{ }^{2} \mathrm{D}_{5 / 2}$ & 3 & 5 & 2 & 5 \\
13 & $n \mathrm{f}{ }^{2} \mathrm{~F}_{7 / 2}^{\mathrm{o}}$ & - & $3 \mathrm{~d}{ }^{2} \mathrm{D}_{5 / 2}$ & 3 & 7 & 2 & 5 \\
\hline
\end{tabular}

The density values range from a typical $\mathrm{H}$ II region value of $100 \mathrm{~cm}^{-3}$ to a value typical of the ionized gas around symbiotic stars.

In Table 2 we define an index for each line component. For transitions from $n \rightarrow 2$ there are seven components, and for $n \rightarrow 3$ there are thirteen. The states involved in the transition are described by their values of $\ell$ and $j$.

The indices defined in Tables $2 \mathrm{a}$ and $2 \mathrm{~b}$ are used to identify the line components in our principal set of results. These are listed in Tables $3 \mathrm{a}-\mathrm{g}$ for Baker \& Menzel's Case B, and in Tables $4 \mathrm{a}-\mathrm{g}$ for Case A. The full tables are only available in electronic form. Here we present only Tables $3 \mathrm{a}$ and $4 \mathrm{a}$. For each line, the reference wavelength (defined in Sect. 2) defines zero velocity for the line components. The shift in wavelength $(\Delta \lambda)$ and in velocity $(\Delta v)$ is listed for each component. Normalized intensities (which sum to unity over the vertical columns of Tables 3 and 4) are listed for the adopted ranges of electron temperature and density for $\mathrm{H}$ I and He II.

It must be emphasized that the reference wavelength does not represent any mean wavelength for the line in question, but is simply a reference point to anchor our chosen scale of velocity shifts. Most of the shifts are negative for the reasons given in Sect. 2.

Tables 5a-d show our computed emissivities for $n_{\mathrm{u}} \rightarrow$ $n_{1}$ transitions, in units of $\mathrm{erg} \mathrm{cm}^{3} \mathrm{~s}^{-1}$, for the full range of electron densities and temperatures considered. From these, the absolute emissivity of any component listed in Tables 3 and 4 can be computed: the normalized intensities listed there should be multiplied by the total emissivity from Table 5. 
Table 3. Normalized component intensities for $\mathrm{H} \alpha: \mathrm{H} 3 \rightarrow 2 ; \lambda_{\text {ref }}=6562.8812 \AA$; Case B

\begin{tabular}{|c|c|c|c|c|c|c|c|c|}
\hline \multirow{2}{*}{$\begin{array}{c}N_{\mathrm{e}} \\
{\left[\mathrm{cm}^{-3}\right]}\end{array}$} & \multirow[b]{2}{*}{ Index } & \multirow{2}{*}{$\begin{array}{c}\Delta \lambda \\
{[\AA]}\end{array}$} & \multirow{2}{*}{$\begin{array}{c}\Delta v \\
{\left[\mathrm{~km} \mathrm{~s}^{-1}\right]}\end{array}$} & \multicolumn{5}{|c|}{$T[\mathrm{~K}]$} \\
\hline & & & & 300 & 1000 & 3000 & 10000 & 30000 \\
\hline \multirow[t]{7}{*}{$10^{2}$} & 1 & -.130 & -5.93 & .018 & .023 & .031 & .044 & .061 \\
\hline & 2 & .028 & 1.27 & .036 & .046 & .061 & .088 & .121 \\
\hline & 3 & -.110 & -5.03 & .057 & .069 & .085 & .106 & .125 \\
\hline & 4 & -.157 & -7.16 & .113 & .138 & .170 & .213 & .250 \\
\hline & 5 & -.172 & -7.85 & .259 & .241 & .218 & .183 & .148 \\
\hline & 6 & -.014 & -0.65 & .052 & .048 & .044 & .037 & .030 \\
\hline & 7 & -.030 & -1.36 & .465 & .435 & .392 & .330 & .266 \\
\hline \multirow[t]{7}{*}{$10^{4}$} & 1 & -.130 & -5.93 & .021 & .025 & .032 & .044 & .061 \\
\hline & 2 & .028 & 1.27 & .042 & .049 & .063 & .088 & .121 \\
\hline & 3 & -.110 & -5.03 & .064 & .073 & .087 & .107 & .125 \\
\hline & 4 & -.157 & -7.16 & .129 & .147 & .175 & .215 & .250 \\
\hline & 5 & -.172 & -7.85 & .248 & .235 & .214 & .182 & .147 \\
\hline & 6 & -.014 & -0.65 & .050 & .047 & .043 & .036 & .029 \\
\hline & 7 & -.030 & -1.36 & .447 & .423 & .386 & .328 & .265 \\
\hline \multirow[t]{7}{*}{$10^{6}$} & 1 & -.130 & -5.93 & .026 & .028 & .034 & .045 & .060 \\
\hline & 2 & .028 & 1.27 & .052 & .057 & .067 & .089 & .121 \\
\hline & 3 & -.110 & -5.03 & .079 & .084 & .094 & .110 & .126 \\
\hline & 4 & -.157 & -7.16 & .159 & .168 & .187 & .220 & .252 \\
\hline & 5 & -.172 & -7.85 & .228 & .221 & .206 & .179 & .147 \\
\hline & 6 & -.014 & -0.65 & .046 & .044 & .041 & .036 & .029 \\
\hline & 7 & -.030 & -1.36 & .410 & .398 & .371 & .322 & .265 \\
\hline \multirow[t]{7}{*}{$10^{7}$} & 1 & -.130 & -5.93 & .029 & .031 & .035 & .045 & .060 \\
\hline & 2 & .028 & 1.27 & .058 & .061 & .070 & .089 & .119 \\
\hline & 3 & -.110 & -5.03 & .090 & .092 & .099 & .113 & .127 \\
\hline & 4 & -.157 & -7.16 & .181 & .185 & .198 & .225 & .253 \\
\hline & 5 & -.172 & -7.85 & .214 & .210 & .199 & .176 & .147 \\
\hline & 6 & -.014 & -0.65 & .043 & .042 & .040 & .035 & .029 \\
\hline & 7 & -.030 & -1.36 & .385 & .379 & .359 & .317 & .265 \\
\hline \multirow[t]{7}{*}{$10^{8}$} & 1 & -.130 & -5.93 & .025 & .027 & .032 & .041 & .055 \\
\hline & 2 & .028 & 1.27 & .050 & .055 & .063 & .081 & .110 \\
\hline & 3 & -.110 & -5.03 & .106 & .105 & .109 & .118 & .130 \\
\hline & 4 & -.157 & -7.16 & .211 & .210 & .217 & .237 & .260 \\
\hline & 5 & -.172 & -7.85 & .203 & .201 & .193 & .174 & .149 \\
\hline & 6 & -.014 & -0.65 & .041 & .040 & .039 & .035 & .030 \\
\hline & 7 & -.030 & -1.36 & .365 & .361 & .347 & .314 & .268 \\
\hline \multirow[t]{7}{*}{$10^{9}$} & 1 & -.130 & -5.93 & .014 & .016 & .019 & .025 & .035 \\
\hline & 2 & .028 & 1.27 & .028 & .032 & .037 & .049 & .070 \\
\hline & 3 & -.110 & -5.03 & .103 & .107 & .113 & .124 & .137 \\
\hline & 4 & -.157 & -7.16 & .206 & .213 & .226 & .248 & .275 \\
\hline & 5 & -.172 & -7.85 & .216 & .211 & .202 & .185 & .161 \\
\hline & 6 & -.014 & -0.65 & .043 & .042 & .040 & .037 & .032 \\
\hline & 7 & -.030 & -1.36 & .390 & .379 & .363 & .332 & .290 \\
\hline
\end{tabular}


Table 4. Normalized component intensities for $\mathrm{H} \alpha$ : $\mathrm{H} 3 \rightarrow 2 ; \lambda_{\text {ref }}=6562.8812 \AA$; Case A

\begin{tabular}{|c|c|c|c|c|c|c|c|c|}
\hline \multirow{2}{*}{$\begin{array}{c}N_{\mathrm{e}} \\
{\left[\mathrm{cm}^{-3}\right]}\end{array}$} & \multirow[b]{2}{*}{ Index } & \multirow{2}{*}{$\begin{array}{c}\Delta \lambda \\
{[\AA]}\end{array}$} & \multirow{2}{*}{$\begin{array}{c}\Delta v \\
{\left[\mathrm{~km} \mathrm{~s}^{-1}\right]}\end{array}$} & \multicolumn{5}{|c|}{$T[\mathrm{~K}]$} \\
\hline & & & & 300 & 1000 & 3000 & 10000 & 30000 \\
\hline \multirow[t]{7}{*}{$10^{2}$} & 1 & -.130 & -5.93 & .012 & .016 & .024 & .040 & .066 \\
\hline & 2 & .028 & 1.27 & .024 & .032 & .048 & .080 & .132 \\
\hline & 3 & -.110 & -5.03 & .008 & .010 & .013 & .018 & .024 \\
\hline & 4 & -.157 & -7.16 & .016 & .020 & .027 & .037 & .048 \\
\hline & 5 & -.172 & -7.85 & .314 & .307 & .296 & .275 & .243 \\
\hline & 6 & -.014 & -0.65 & .063 & .061 & .059 & .055 & .049 \\
\hline & 7 & -.030 & -1.36 & .564 & .553 & .533 & .495 & .438 \\
\hline \multirow[t]{7}{*}{$10^{4}$} & 1 & -.130 & -5.93 & .014 & .018 & .025 & .041 & .067 \\
\hline & 2 & .028 & 1.27 & .028 & .036 & .050 & .082 & .134 \\
\hline & 3 & -.110 & -5.03 & .009 & .011 & .014 & .019 & .024 \\
\hline & 4 & -.157 & -7.16 & .019 & .022 & .028 & .038 & .048 \\
\hline & 5 & -.172 & -7.85 & .310 & .305 & .294 & .274 & .242 \\
\hline & 6 & -.014 & -0.65 & .062 & .061 & .059 & .055 & .048 \\
\hline & 7 & -.030 & -1.36 & .558 & .548 & .530 & .493 & .436 \\
\hline \multirow[t]{7}{*}{$10^{6}$} & 1 & -.130 & -5.93 & .020 & .022 & .029 & .043 & .069 \\
\hline & 2 & .028 & 1.27 & .040 & .045 & .057 & .087 & .137 \\
\hline & 3 & -.110 & -5.03 & .012 & .013 & .015 & .020 & .024 \\
\hline & 4 & -.157 & -7.16 & .024 & .026 & .031 & .039 & .049 \\
\hline & 5 & -.172 & -7.85 & .301 & .298 & .289 & .270 & .240 \\
\hline & 6 & -.014 & -0.65 & .060 & .060 & .058 & .054 & .048 \\
\hline & 7 & -.030 & -1.36 & .543 & .536 & .521 & .487 & .433 \\
\hline \multirow[t]{7}{*}{$10^{7}$} & 1 & -.130 & -5.93 & .024 & .026 & .032 & .046 & .070 \\
\hline & 2 & .028 & 1.27 & .048 & .052 & .063 & .091 & .140 \\
\hline & 3 & -.110 & -5.03 & .014 & .015 & .017 & .020 & .025 \\
\hline & 4 & -.157 & -7.16 & .029 & .030 & .033 & .040 & .049 \\
\hline & 5 & -.172 & -7.85 & .295 & .292 & .285 & .267 & .239 \\
\hline & 6 & -.014 & -0.65 & .059 & .058 & .057 & .053 & .048 \\
\hline & 7 & -.030 & -1.36 & .531 & .526 & .513 & .481 & .430 \\
\hline \multirow[t]{7}{*}{$10^{8}$} & 1 & -.130 & -5.93 & .023 & .026 & .032 & .045 & .069 \\
\hline & 2 & .028 & 1.27 & .046 & .052 & .063 & .090 & .137 \\
\hline & 3 & -.110 & -5.03 & .018 & .018 & .019 & .022 & .025 \\
\hline & 4 & -.157 & -7.16 & .037 & .036 & .038 & .044 & .051 \\
\hline & 5 & -.172 & -7.85 & .292 & .289 & .283 & .266 & .239 \\
\hline & 6 & -.014 & -0.65 & .058 & .058 & .057 & .053 & .048 \\
\hline & 7 & -.030 & -1.36 & .526 & .520 & .509 & .479 & .431 \\
\hline \multirow[t]{7}{*}{$10^{9}$} & 1 & -.130 & -5.93 & .011 & .014 & .018 & .028 & .047 \\
\hline & 2 & .028 & 1.27 & .023 & .027 & .036 & .056 & .094 \\
\hline & 3 & -.110 & -5.03 & .027 & .026 & .026 & .028 & .032 \\
\hline & 4 & -.157 & -7.16 & .054 & .052 & .052 & .056 & .063 \\
\hline & 5 & -.172 & -7.85 & .295 & .294 & .289 & .277 & .255 \\
\hline & 6 & -.014 & -0.65 & .059 & .059 & .058 & .055 & .051 \\
\hline & 7 & -.030 & -1.36 & .531 & .529 & .521 & .499 & .458 \\
\hline
\end{tabular}


Table 5a. H I line emissivities $\left[\mathrm{erg}_{\mathrm{cm}}^{3} \mathrm{~s}^{-1}\right]$ : Case B

\begin{tabular}{ccrrrrr}
\hline & $N_{\mathrm{e}}$ & \multicolumn{5}{c}{$T[\mathrm{~K}]$} \\
\cline { 3 - 6 }$n_{\mathrm{u}}-n_{\mathrm{l}}$ & {$\left[\mathrm{cm}^{-3}\right]$} & 300 & 1000 & 3000 & 10000 & 30000 \\
\hline $3-2$ & $10^{2}$ & $6.796(-24)$ & $2.617(-24)$ & $1.048(-24)$ & $3.537(-25)$ & $1.199(-25)$ \\
& $10^{4}$ & $7.440(-24)$ & $2.655(-24)$ & $1.047(-24)$ & $3.530(-25)$ & $1.199(-25)$ \\
& $10^{6}$ & $1.026(-23)$ & $2.859(-24)$ & $1.057(-24)$ & $3.522(-25)$ & $1.198(-25)$ \\
& $10^{7}$ & $1.472(-23)$ & $3.174(-24)$ & $1.081(-24)$ & $3.526(-25)$ & $1.197(-25)$ \\
& $10^{8}$ & $2.660(-23)$ & $3.894(-24)$ & $1.144(-24)$ & $3.557(-25)$ & $1.198(-25)$ \\
& $10^{9}$ & $6.754(-23)$ & $5.703(-24)$ & $1.309(-24)$ & $3.685(-25)$ & $1.211(-25)$ \\
\hline $4-2$ & $10^{2}$ & $1.613(-24)$ & $7.146(-25)$ & $3.265(-25)$ & $1.235(-25)$ & $4.440(-26)$ \\
& $10^{4}$ & $1.959(-24)$ & $7.623(-25)$ & $3.331(-25)$ & $1.240(-25)$ & $4.443(-26)$ \\
& $10^{6}$ & $3.125(-24)$ & $8.974(-25)$ & $3.512(-25)$ & $1.255(-25)$ & $4.450(-26)$ \\
& $10^{7}$ & $4.849(-24)$ & $1.055(-24)$ & $3.712(-25)$ & $1.272(-25)$ & $4.459(-26)$ \\
& $10^{8}$ & $9.658(-24)$ & $1.401(-24)$ & $4.143(-25)$ & $1.317(-25)$ & $4.514(-26)$ \\
& $10^{9}$ & $2.666(-23)$ & $2.227(-24)$ & $5.049(-25)$ & $1.409(-25)$ & $4.625(-26)$ \\
\hline $5-2$ & $10^{2}$ & $6.677(-25)$ & $3.078(-25)$ & $1.469(-25)$ & $5.784(-26)$ & $2.123(-26)$ \\
& $10^{4}$ & $8.296(-25)$ & $3.323(-25)$ & $1.506(-25)$ & $5.818(-26)$ & $2.124(-26)$ \\
& $10^{6}$ & $1.394(-24)$ & $4.042(-25)$ & $1.614(-25)$ & $5.917(-26)$ & $2.128(-26)$ \\
& $10^{7}$ & $2.300(-24)$ & $4.969(-25)$ & $1.753(-25)$ & $6.072(-26)$ & $2.144(-26)$ \\
& $10^{8}$ & $5.056(-24)$ & $7.188(-25)$ & $2.079(-25)$ & $6.487(-26)$ & $2.206(-26)$ \\
& $10^{9}$ & $1.488(-23)$ & $1.223(-24)$ & $2.674(-25)$ & $7.038(-26)$ & $2.205(-26)$ \\
\hline
\end{tabular}

Table 5b. He II line emissivities $\left[\mathrm{erg} \mathrm{cm}^{3} \mathrm{~s}^{-1}\right.$ ]: Case B

\begin{tabular}{ccrrrr}
\hline & $N_{\mathrm{e}}$ & \multicolumn{4}{c}{$T[\mathrm{~K}]$} \\
\cline { 3 - 6 }$n_{\mathrm{u}}-n_{\mathrm{l}}$ & {$\left[\mathrm{cm}^{-3}\right]$} & 1000 & 3000 & 10000 & 30000 \\
\hline $3-2$ & $10^{4}$ & $6.263(-23)$ & $2.630(-23)$ & $9.790(-24)$ & $3.701(-24)$ \\
& $10^{6}$ & $7.097(-23)$ & $2.701(-23)$ & $9.790(-24)$ & $3.692(-24)$ \\
& $10^{7}$ & $8.268(-23)$ & $2.811(-23)$ & $9.832(-24)$ & $3.687(-24)$ \\
& $10^{8}$ & $1.086(-22)$ & $3.049(-23)$ & $9.962(-24)$ & $3.687(-24)$ \\
& $10^{9}$ & $1.733(-22)$ & $3.575(-23)$ & $1.030(-23)$ & $3.702(-24)$ \\
\hline $4-2$ & $10^{4}$ & $1.459(-23)$ & $6.939(-24)$ & $2.991(-24)$ & $1.264(-24)$ \\
& $10^{6}$ & $1.842(-23)$ & $7.557(-24)$ & $3.063(-24)$ & $1.271(-24)$ \\
& $10^{7}$ & $2.308(-23)$ & $8.231(-24)$ & $3.140(-24)$ & $1.280(-24)$ \\
& $10^{8}$ & $3.277(-23)$ & $9.431(-24)$ & $3.270(-24)$ & $1.293(-24)$ \\
& $10^{9}$ & $5.657(-23)$ & $1.176(-23)$ & $3.504(-24)$ & $1.319(-24)$ \\
\hline $4-3$ & $10^{4}$ & $1.263(-23)$ & $4.766(-24)$ & $1.492(-24)$ & $4.623(-25)$ \\
& $10^{6}$ & $1.309(-23)$ & $4.612(-24)$ & $1.444(-24)$ & $4.536(-25)$ \\
& $10^{7}$ & $1.418(-23)$ & $4.554(-24)$ & $1.406(-24)$ & $4.459(-25)$ \\
& $10^{8}$ & $1.699(-23)$ & $4.600(-24)$ & $1.362(-24)$ & $4.356(-25)$ \\
& $10^{9}$ & $2.434(-23)$ & $4.935(-24)$ & $1.327(-24)$ & $4.232(-25)$ \\
\hline $5-3$ & $10^{4}$ & $3.825(-24)$ & $1.662(-24)$ & $6.149(-25)$ & $2.169(-25)$ \\
& $10^{6}$ & $4.551(-24)$ & $1.741(-24)$ & $6.166(-25)$ & $2.161(-25)$ \\
& $10^{7}$ & $5.381(-24)$ & $1.820(-24)$ & $6.177(-25)$ & $2.152(-25)$ \\
& $10^{8}$ & $7.066(-24)$ & $1.965(-24)$ & $6.205(-25)$ & $2.136(-25)$ \\
& $10^{9}$ & $1.114(-23)$ & $2.271(-24)$ & $6.315(-25)$ & $2.117(-25)$ \\
\hline
\end{tabular}


Table 5c. H I line emissivities $\left[\mathrm{erg} \mathrm{cm}^{3} \mathrm{~s}^{-1}\right]$ : Case A

\begin{tabular}{ccrrrrr}
\hline & $N_{\mathrm{e}}$ & \multicolumn{5}{c}{$T[\mathrm{~K}]$} \\
\cline { 3 - 7 }$n_{\mathrm{u}-n_{\mathrm{l}}}\left[\mathrm{cm}^{-3}\right]$ & 300 & 1000 & 3000 & 10000 & 30000 \\
\hline $3-2$ & $10^{2}$ & $5.494(-24)$ & $2.031(-24)$ & $7.615(-25)$ & $2.327(-25)$ & $7.160(-26)$ \\
& $10^{4}$ & $5.670(-24)$ & $1.988(-24)$ & $7.449(-25)$ & $2.297(-25)$ & $7.119(-26)$ \\
& $10^{6}$ & $6.845(-24)$ & $1.958(-24)$ & $7.102(-25)$ & $2.220(-25)$ & $6.998(-26)$ \\
& $10^{7}$ & $8.777(-24)$ & $2.004(-24)$ & $6.872(-25)$ & $2.151(-25)$ & $6.874(-26)$ \\
& $10^{8}$ & $1.338(-23)$ & $2.155(-24)$ & $6.609(-25)$ & $2.039(-25)$ & $6.611(-26)$ \\
& $10^{9}$ & $2.635(-23)$ & $2.533(-24)$ & $6.308(-25)$ & $1.827(-25)$ & $5.903(-26)$ \\
\hline $4-2$ & $10^{2}$ & $1.220(-24)$ & $5.302(-25)$ & $2.331(-25)$ & $8.246(-26)$ & $2.758(-26)$ \\
& $10^{4}$ & $1.425(-24)$ & $5.536(-25)$ & $2.350(-25)$ & $8.235(-26)$ & $2.754(-26)$ \\
& $10^{6}$ & $2.052(-24)$ & $6.103(-25)$ & $2.381(-25)$ & $8.162(-26)$ & $2.732(-26)$ \\
& $10^{7}$ & $2.874(-24)$ & $6.669(-25)$ & $2.394(-25)$ & $8.030(-26)$ & $2.691(-26)$ \\
& $10^{8}$ & $4.846(-24)$ & $7.723(-25)$ & $2.399(-25)$ & $7.659(-26)$ & $2.549(-26)$ \\
& $10^{9}$ & $1.105(-23)$ & $1.036(-24)$ & $2.527(-25)$ & $7.237(-26)$ & $2.317(-26)$ \\
\hline $5-2$ & $10^{2}$ & $4.925(-25)$ & $2.248(-25)$ & $1.044(-25)$ & $3.903(-26)$ & $1.348(-26)$ \\
& $10^{4}$ & $5.928(-25)$ & $2.387(-25)$ & $1.061(-25)$ & $3.910(-26)$ & $1.347(-26)$ \\
& $10^{6}$ & $9.071(-25)$ & $2.737(-25)$ & $1.098(-25)$ & $3.904(-26)$ & $1.338(-26)$ \\
& $10^{7}$ & $1.344(-24)$ & $3.102(-25)$ & $1.124(-25)$ & $3.846(-26)$ & $1.308(-26)$ \\
& $10^{8}$ & $2.550(-24)$ & $3.958(-25)$ & $1.197(-25)$ & $3.741(-26)$ & $1.227(-26)$ \\
& $10^{9}$ & $6.688(-24)$ & $6.100(-25)$ & $1.423(-25)$ & $3.852(-26)$ & $1.189(-26)$ \\
\hline
\end{tabular}

Table 5d. He II line emissivities $\left[\mathrm{erg} \mathrm{cm}^{3} \mathrm{~s}^{-1}\right.$ ]: Case A

\begin{tabular}{ccrrrr}
\hline \multicolumn{5}{c}{$N_{\mathrm{e}}$} & \multicolumn{4}{c}{$T[\mathrm{~K}]$} \\
\cline { 3 - 6 }$n_{\mathrm{u}}-n_{\mathrm{l}}$ & {$\left[\mathrm{cm}^{-3}\right]$} & 1000 & 3000 & 10000 & 30000 \\
\hline $3-2$ & $10^{4}$ & $5.079(-23)$ & $2.064(-23)$ & $7.197(-24)$ & $2.496(-24)$ \\
& $10^{6}$ & $5.417(-23)$ & $2.039(-23)$ & $7.035(-24)$ & $2.456(-24)$ \\
& $10^{7}$ & $5.969(-23)$ & $2.044(-23)$ & $6.904(-24)$ & $2.420(-24)$ \\
& $10^{8}$ & $7.237(-23)$ & $2.093(-23)$ & $6.745(-24)$ & $2.367(-24)$ \\
& $10^{9}$ & $1.030(-22)$ & $2.253(-23)$ & $6.589(-24)$ & $2.293(-24)$ \\
\hline \multirow{2}{*}{$4-2$} & $10^{4}$ & $1.104(-23)$ & $5.181(-24)$ & $2.152(-24)$ & $8.590(-25)$ \\
& $10^{6}$ & $1.340(-23)$ & $5.509(-24)$ & $2.175(-24)$ & $8.581(-25)$ \\
& $10^{7}$ & $1.611(-23)$ & $5.844(-24)$ & $2.196(-24)$ & $8.565(-25)$ \\
& $10^{8}$ & $2.146(-23)$ & $6.407(-24)$ & $2.227(-24)$ & $8.525(-25)$ \\
& $10^{9}$ & $3.341(-23)$ & $7.403(-24)$ & $2.269(-24)$ & $8.417(-25)$ \\
\hline $4-3$ & $10^{4}$ & $1.205(-23)$ & $4.535(-24)$ & $1.397(-24)$ & $4.192(-25)$ \\
& $10^{6}$ & $1.201(-23)$ & $4.278(-24)$ & $1.328(-24)$ & $4.056(-25)$ \\
& $10^{7}$ & $1.254(-23)$ & $4.118(-24)$ & $1.270(-24)$ & $3.935(-25)$ \\
& $10^{8}$ & $1.419(-23)$ & $3.994(-24)$ & $1.196(-24)$ & $3.763(-25)$ \\
& $10^{9}$ & $1.862(-23)$ & $4.013(-24)$ & $1.112(-24)$ & $3.528(-25)$ \\
\hline $5-3$ & $10^{4}$ & $3.572(-24)$ & $1.549(-24)$ & $5.634(-25)$ & $1.925(-25)$ \\
& $10^{6}$ & $4.101(-24)$ & $1.587(-24)$ & $5.573(-25)$ & $1.900(-25)$ \\
& $10^{7}$ & $4.678(-24)$ & $1.620(-24)$ & $5.499(-25)$ & $1.872(-25)$ \\
& $10^{8}$ & $5.802(-24)$ & $1.680(-24)$ & $5.378(-25)$ & $1.824(-25)$ \\
& $10^{9}$ & $8.323(-24)$ & $1.808(-24)$ & $5.197(-25)$ & $1.738(-25)$ \\
\hline
\end{tabular}


Where comparison can be made, our emissivities for $\mathrm{HI}$ and $\mathrm{HII}$, extrapolated to zero electron density, are in good agreement with those of Martin (1988). Differences are always less than two percent.

The highest temperature for which results are given for $\mathrm{HI}$ is $30000 \mathrm{~K}$. At this temperature, and with the hydrogen ionization fractions typical of gaseous nebulae, there will be significant collisional excitation from the ground state to the low-lying states of interest in this work. The values given in the tables for $30000 \mathrm{~K}$ were calculated assuming that there is no such collisional excitation, and should therefore only be used if a) it is known that the fraction of neutral hydrogen is very low (see HS for the permitted range of values), or b) it is required to interpolate the tables to some intermediate temperature. Collisional excitation from the ground state is negligible at the next-lowest tabulated temperature $(10000 \mathrm{~K})$.

\section{Applications and discussion}

\subsection{Appearance of the $\mathrm{H}$ and $\mathrm{He}$ lines}

Figures $1 \mathrm{a}-\mathrm{c}$ show the components and their predicted intensities for the three selected lines $\mathrm{H} \alpha, \mathrm{H} \beta$ and $\mathrm{H} \gamma$. In the figures, the vertical bars have a length proportional to the relative intensity of the component for the Case B, $T_{\mathrm{e}}=5000 \mathrm{~K}$ and $N_{\mathrm{e}}=10^{4} \mathrm{~cm}^{-3}$. The smooth curves represent a convolution of these intensities with the thermal velocity expected in a $5000 \mathrm{~K}$ plasma for $\mathrm{H}$ $\left(F W H M=15.1 \mathrm{~km} \mathrm{~s}^{-1}\right)$. They are however arbitrarily normalized, for presentation purposes. The HI Balmer lines each have seven components, with the total velocity range shrinking from 9.1 to $5.0 \mathrm{~km} \mathrm{~s}^{-1}$ between $\mathrm{H} \alpha$ and $\mathrm{H} \gamma$. For $\mathrm{H} \alpha$, the convolved profile is double-peaked if the total broadening velocity is not more than $5 \mathrm{~km} \mathrm{~s}^{-1}$; it is single but noticeably non-Gaussian for $7 \mathrm{~km} \mathrm{~s}^{-1}$, and is difficult to distinguish from Gaussian for a total velocity over $11 \mathrm{~km} \mathrm{~s}^{-1}$.

Figures $2 \mathrm{a}-\mathrm{d}$ show the components and their predicted intensities for the four selected lines of $\mathrm{He}$ for the Case B, $T_{\mathrm{e}}=10000 \mathrm{~K}$ and $N_{\mathrm{e}}=10^{4} \mathrm{~cm}^{-3}$. A convolution of these intensities was calculated for a $10000 \mathrm{~K}$ plasma $\left(F W H M=10.7 \mathrm{~km} \mathrm{~s}^{-1}\right)$.

At a given plasma temperature, the component spread is four times greater and the thermal velocity is two times smaller. He II $4686 \AA$ is mainly broadened by the effect of two strong components situated $6.42 \mathrm{~km} \mathrm{~s}^{-1}$ apart. As can be seen from Fig. 2a, the weak components in the blue part of the line profile should not be interpreted as a blue-shifted emission line gas moving at $-25 \mathrm{~km} \mathrm{~s}^{-1}$ ! The effect on the $n=5 \rightarrow 3$ line at $3203 \AA$ is quite similar (Fig. 2b).

However, there are larger effects on the $3 \rightarrow 2$ and $4 \rightarrow 2$ lines in the ultraviolet (Figs. 2c,d). The $1640 \AA$ and $1215 \AA$ lines are clearly doubled when the only broadening is thermal, with peak-to-peak splittings of about 25 and $20 \mathrm{~km} \mathrm{~s}^{-1}$ respectively.
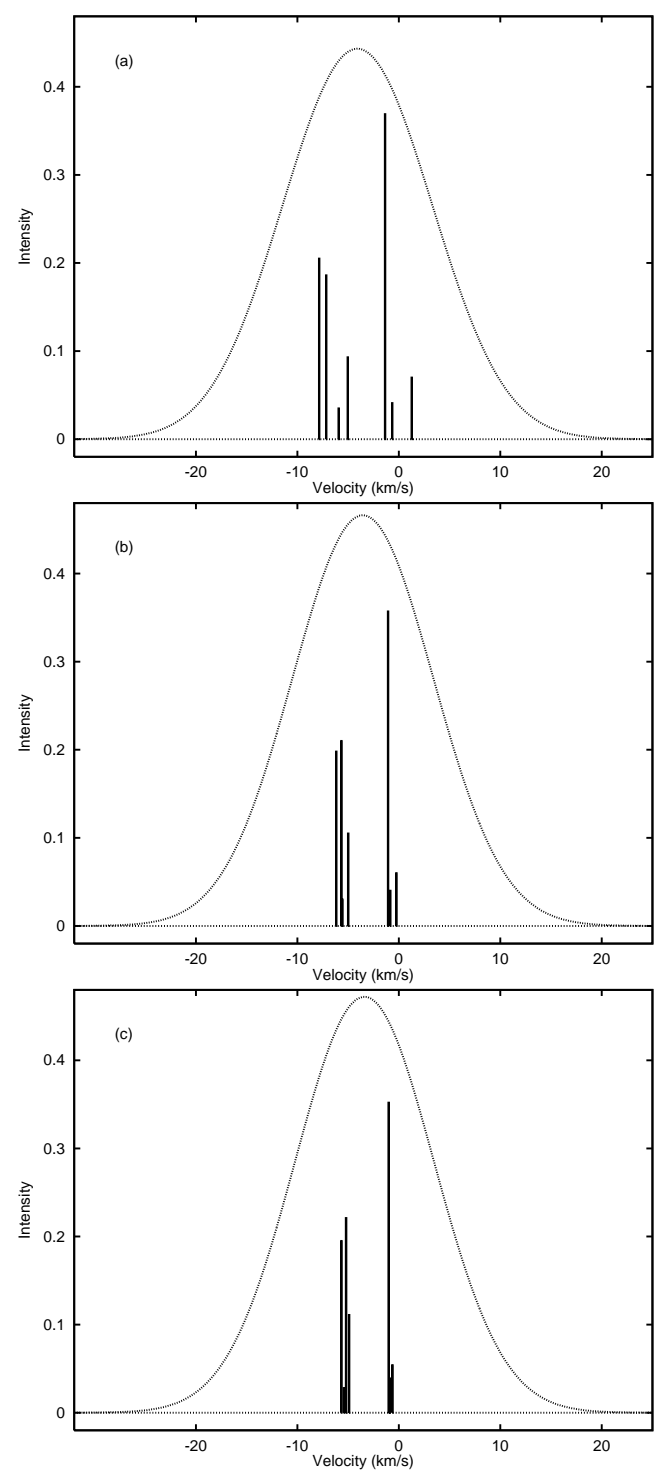

Fig. 1. Profiles of the H I emission lines in velocity space: a) $\mathrm{H} \alpha, \mathbf{b})-\mathrm{H} \beta, \mathbf{c})-\mathrm{H} \gamma$. The vertical bars show the position and the relative intensity of the seven components of these lines for the Case B, $T_{\mathrm{e}}=5000 \mathrm{~K}$ and $N_{\mathrm{e}}=10^{4} \mathrm{~cm}^{-3}$. The smooth curve shows these intensities convolved with a velocity of $15.1 \mathrm{~km} \mathrm{~s}^{-1}$, the $F W H M$ for thermal broadening of $\mathrm{H}$ at $5000 \mathrm{~K}$

He II widths may be used to measure the velocity gradients within expanding planetary nebulae. The expansion velocity in the outer regions may be measured from $[\mathrm{O} \mathrm{I}],[\mathrm{O} \mathrm{II}]$ or $[\mathrm{N} \mathrm{II}]$ lines, in the central region from [O III] lines, and in the inner zones from HeII lines such as 1640 or $4686 \AA$. Correction of the He II line widths for fine-structure will tend to steepen the derived gradient of expansion velocity with radius.

\subsection{Line shifts}

Accurate line centre positions are required both for precision radial velocity measurements and also for accurate 

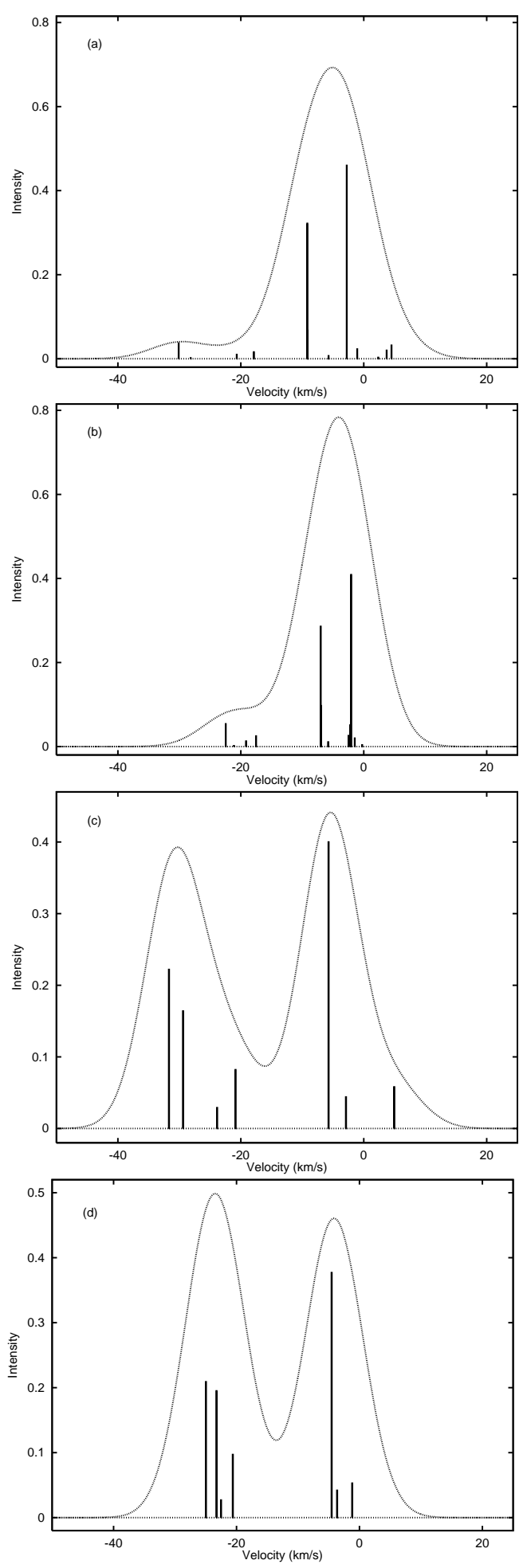

Fig. 2. Profiles of the He II emission lines in velocity space for the Case B, $T_{\mathrm{e}}=10000 \mathrm{~K}$ and $N_{\mathrm{e}}=10^{4} \mathrm{~cm}^{-3}$ : a) - He II $4686 \AA$ line, b) - He II $3203 \AA$ line, c) - He II $1215 \AA$ line, d) - He II $1640 \AA$ line. The smooth curve shows the component intensities convolved with a velocity of $10.7 \mathrm{~km} \mathrm{~s}^{-1}$, the FWHM for thermal broadening of $\mathrm{He}$ at $10000 \mathrm{~K}$
Table 6a. Line centre positions for H I (Case B). Entries show the decimal part of the mean wavelength, in $\AA$

\begin{tabular}{ccccccc}
\hline & $N_{\mathrm{e}}$ & \multicolumn{5}{c}{$T[\mathrm{~K}]$} \\
\cline { 3 - 7 }$n_{\mathrm{u}}-n_{\mathrm{l}}\left[\mathrm{cm}^{-3}\right]$ & 300 & 1000 & 3000 & 10000 & 30000 \\
\hline $3-2$ & $10^{2}$ & .7969 & .7954 & .7930 & .7910 & .7900 \\
& $10^{4}$ & .7959 & .7944 & .7930 & .7910 & .7900 \\
6562 & $10^{6}$ & .7935 & .7930 & .7920 & .7905 & .7900 \\
& $10^{7}$ & .7920 & .7920 & .7910 & .7900 & .7896 \\
& $10^{8}$ & .7881 & .7891 & .7886 & .7881 & .7881 \\
& $10^{9}$ & .7871 & .7866 & .7856 & .7847 & .7837 \\
\hline $4-2$ & $10^{2}$ & .3237 & .3232 & .3218 & .3198 & .3184 \\
& $10^{4}$ & .3237 & .3228 & .3218 & .3198 & .3184 \\
4861 & $10^{6}$ & .3223 & .3223 & .3213 & .3198 & .3184 \\
& $10^{7}$ & .3213 & .3213 & .3208 & .3193 & .3179 \\
& $10^{8}$ & .3208 & .3208 & .3198 & .3188 & .3174 \\
& $10^{9}$ & .3237 & .3232 & .3228 & .3213 & .3188 \\
\hline $5-2$ & $10^{2}$ & .4619 & .4609 & .4604 & .4590 & .4575 \\
& $10^{4}$ & .4614 & .4609 & .4604 & .4590 & .4575 \\
4340 & $10^{6}$ & .4609 & .4604 & .4600 & .4590 & .4575 \\
& $10^{7}$ & .4604 & .4604 & .4600 & .4585 & .4570 \\
& $10^{8}$ & .4624 & .4619 & .4609 & .4595 & .4580 \\
& $10^{9}$ & .4629 & .4629 & .4629 & .4624 & .4614 \\
\hline \multirow{4}{*}{54}
\end{tabular}

calibration of Fabry-Perot high-resolution interferometers (Treffers 1981).

We have thus computed the "centres of gravity" of the emission lines we considered. These are calculated simply as the mean position of all the line components, each weighted by its relative intensity for the Case and physical conditions considered. This will correspond to the measured position of an emission line when it is completely unresolved. The mean wavelengths of the lines are given in Tables 6a-d; the tables show the decimal part of the mean wavelength in $\AA$, with the integer part presented separately in the first column of the Table. Wavelengths are in air for $\lambda>2000 \AA$ and in vacuum otherwise.

There are shifts in the line centres which are relevant for high-precision measurement of radial velocities or velocity fields. For a given Case (A or B) the maximum line centre shift with varying $N_{\mathrm{e}}$ or $T_{\mathrm{e}}$ is $0.6 \mathrm{~km} \mathrm{~s}^{-1}$ for hydrogen $\left(\mathrm{H} \alpha\right.$, Case B) and $1.3 \mathrm{~km} \mathrm{~s}^{-1}$ for helium $(\lambda 1640 \AA$, Case B). The largest shifts of one line between Case A and Case B are $0.9 \mathrm{~km} \mathrm{~s}^{-1}$ for $\mathrm{H}(\mathrm{H} \alpha)$ and $3.3 \mathrm{~km} \mathrm{~s}^{-1}$ for He II $(\lambda 1640 \AA)$.

Our computed shift between Cases A and B for $\mathrm{H} \alpha$ $\left(0.9 \mathrm{~km} \mathrm{~s}^{-1}\right)$ is smaller than the value of $2.5 \mathrm{~km} \mathrm{~s}^{-1}$ reported by Dyson \& Meaburn (1971). The relative intensities given in Table 3a do not differ significantly from the values given by Dyson \& Meaburn (1971) (for $T_{\mathrm{e}}=$ $10000 \mathrm{~K})$. Another possible source of the disagreement 
Table 6b. Line centre positions for He II (Case B). Entries show the decimal part of the mean wavelength, in $\AA$

\begin{tabular}{cccccc}
\hline & $N_{\mathrm{e}}$ & \multicolumn{4}{c}{$T[\mathrm{~K}]$} \\
\cline { 3 - 6 }$n_{\mathrm{u}}-n_{\mathrm{l}}$ & {$\left[\mathrm{cm}^{-3}\right]$} & 1000 & 3000 & 10000 & 30000 \\
\hline $3-2$ & $10^{4}$ & .4196 & .4182 & .4160 & .4138 \\
& $10^{6}$ & .4185 & .4176 & .4156 & .4136 \\
\multirow{2}{*}{1640} & $10^{7}$ & .4175 & .4167 & .4152 & .4133 \\
& $10^{8}$ & .4161 & .4159 & .4144 & .4130 \\
& $10^{9}$ & .4144 & .4143 & .4137 & .4125 \\
\hline \multirow{2}{*}{$4-2$} & $10^{4}$ & .1337 & .1329 & .1317 & .1300 \\
& $10^{6}$ & .1333 & .1327 & .1315 & .1299 \\
1215 & $10^{7}$ & .1329 & .1323 & .1313 & .1299 \\
& $10^{8}$ & .1322 & .1320 & .1310 & .1298 \\
& $10^{9}$ & .1311 & .1310 & .1302 & .1293 \\
\hline $4-3$ & $10^{4}$ & .7534 & .7515 & .7480 & .7446 \\
& $10^{6}$ & .7515 & .7500 & .7471 & .7441 \\
4685 & $10^{7}$ & .7500 & .7480 & .7466 & .7441 \\
& $10^{8}$ & .7476 & .7471 & .7456 & .7437 \\
& $10^{9}$ & .7456 & .7451 & .7437 & .7427 \\
\hline $5-3$ & $10^{4}$ & .1301 & .1282 & .1252 & .1213 \\
& $10^{6}$ & .1287 & .1274 & .1250 & .1211 \\
3203 & $10^{7}$ & .1274 & .1267 & .1243 & .1206 \\
& $10^{8}$ & .1260 & .1255 & .1230 & .1201 \\
& $10^{9}$ & .1233 & .1228 & .1221 & .1191 \\
\hline \multirow{2}{*}{$5-3$}
\end{tabular}

Table 6c. Line centre positions for H I (Case A). Entries show the decimal part of the mean wavelength, in $\AA$

\begin{tabular}{ccccccc}
\hline & $N_{\mathrm{e}}$ & \multicolumn{5}{c}{$T[\mathrm{~K}]$} \\
\cline { 3 - 6 }$n_{\mathrm{u}}-n_{1}\left[\mathrm{~cm}^{-3}\right]$ & 300 & 1000 & 3000 & 10000 & 30000 \\
\hline $3-2$ & $10^{2}$ & .8052 & .8057 & .8062 & .8076 & .8105 \\
& $10^{4}$ & .8052 & .8057 & .8062 & .8076 & .8110 \\
6562 & $10^{6}$ & .8057 & .8062 & .8066 & .8081 & .8110 \\
& $10^{7}$ & .8062 & .8062 & .8066 & .8081 & .8110 \\
& $10^{8}$ & .8052 & .8057 & .8062 & .8076 & .8105 \\
& $10^{9}$ & .8018 & .8018 & .8027 & .8042 & .8062 \\
\hline $4-2$ & $10^{2}$ & .3325 & .3320 & .3320 & .3315 & .3315 \\
& $10^{4}$ & .3320 & .3320 & .3320 & .3315 & .3315 \\
4861 & $10^{6}$ & .3320 & .3320 & .3320 & .3315 & .3315 \\
& $10^{7}$ & .3315 & .3320 & .3315 & .3315 & .3315 \\
& $10^{8}$ & .3301 & .3306 & .3306 & .3306 & .3306 \\
& $10^{9}$ & .3276 & .3281 & .3286 & .3281 & .3276 \\
\hline $5-2$ & $10^{2}$ & .4692 & .4692 & .4692 & .4688 & .4683 \\
& $10^{4}$ & .4692 & .4692 & .4692 & .4688 & .4683 \\
4340 & $10^{6}$ & .4692 & .4692 & .4688 & .4688 & .4683 \\
& $10^{7}$ & .4683 & .4688 & .4688 & .4683 & .4678 \\
& $10^{8}$ & .4668 & .4673 & .4673 & .4668 & .4663 \\
& $10^{9}$ & .4644 & .4648 & .4648 & .4648 & .4644 \\
\hline \multirow{6}{*}{54} & & & & & &
\end{tabular}

Table 6d. Line centre positions for He II (Case A). Entries show the decimal part of the mean wavelength, in $\AA$

\begin{tabular}{cccccc}
\hline & $N_{\mathrm{e}}$ & \multicolumn{4}{c}{$T[\mathrm{~K}]$} \\
\cline { 3 - 6 }$n_{\mathrm{u}}-n_{\mathrm{l}}$ & {$\left[\mathrm{cm}^{-3}\right]$} & 1000 & 3000 & 10000 & 30000 \\
\hline $3-2$ & $10^{4}$ & .4285 & .4285 & .4290 & .4302 \\
& $10^{6}$ & .4283 & .4286 & .4291 & .4301 \\
1640 & $10^{7}$ & .4287 & .4287 & .4291 & .4303 \\
& $10^{8}$ & .4287 & .4288 & .4293 & .4304 \\
& $10^{9}$ & .4290 & .4291 & .4296 & .4307 \\
\hline $4-2$ & $10^{4}$ & .1422 & .1422 & .1418 & .1415 \\
& $10^{6}$ & .1422 & .1421 & .1418 & .1416 \\
1215 & $10^{7}$ & .1422 & .1421 & .1418 & .1416 \\
& $10^{8}$ & .1420 & .1420 & .1417 & .1416 \\
& $10^{9}$ & .1416 & .1416 & .1416 & .1415 \\
\hline $4-3$ & $10^{4}$ & .7529 & .7500 & .7456 & .7412 \\
& $10^{6}$ & .7505 & .7485 & .7446 & .7397 \\
4685 & $10^{7}$ & .7480 & .7466 & .7432 & .7393 \\
& $10^{8}$ & .7456 & .7446 & .7422 & .7388 \\
& $10^{9}$ & .7427 & .7422 & .7402 & .7373 \\
\hline $5-3$ & $10^{4}$ & .1313 & .1296 & .1270 & .1230 \\
& $10^{6}$ & .1301 & .1289 & .1265 & .1228 \\
3203 & $10^{7}$ & .1292 & .1279 & .1260 & .1226 \\
& $10^{8}$ & .1277 & .1270 & .1248 & .1218 \\
& $10^{9}$ & .1252 & .1250 & .1233 & .1208 \\
\hline \multirow{2}{*}{$5-3$} & & & & \\
& & & &
\end{tabular}

could be the data used for the energy levels. We use the results from Erickson (1977) with the Lamb shift included while Dyson \& Meaburn (1971) use relativistic theory but excluding the Lamb shift. The differences in level energies are not, however, sufficient to explain the discrepancy, indicating that the value of $2.5 \mathrm{~km} \mathrm{~s}^{-1}$ given by Dyson \& Meaburn (1971) is in error.

\subsection{The $\mathrm{H} \alpha-[\mathrm{N}$ II $]$ method for deriving electron temperature}

Because the thermal $F W H M$ of an emission line varies with temperature and ionic mass as $T_{\mathrm{e}}^{1 / 2} m^{-1 / 2}$, the electron temperature $T_{\mathrm{e}}$ can in principle be derived from the different observed widths of different ionic lines (Wilson et al. 1959). Observationally the easiest line pair to work with (Courtes et al. 1968) is $\mathrm{H} \alpha$ and $[\mathrm{N} \mathrm{II}] 6584 \AA$, which are only separated by $21 \AA$. The basic assumption is that the emissivity of the two lines samples the velocity field (mainly, expansion and turbulence) in identical ways. While this may be true for some $\mathrm{HII}$ regions, it is frequently untrue for planetary nebulae, where $\mathrm{N}^{+}$ions are often only located at the outer edge.

Meaburn (1970) showed that the fine structure of $\mathrm{H} \alpha$ would affect the resulting derived electron temperature, and Dyson \& Meaburn (1971) gave correction factors to be 
Table 7. $F W H M$ and broadening term $\delta\left[\mathrm{km} \mathrm{s}^{-1}\right]$ for $\mathrm{H} \alpha$

\begin{tabular}{rrr}
\hline$v_{\text {broad }}$ & $F W H M$ & \multicolumn{1}{c}{$\delta$} \\
\hline & & \\
3.0 & 9.41 & 8.914 \\
3.5 & 9.89 & 9.245 \\
4.0 & 10.32 & 9.514 \\
4.5 & 10.73 & 9.739 \\
5.0 & 11.10 & 9.910 \\
6.0 & 11.70 & 10.049 \\
7.0 & 12.09 & 9.860 \\
8.0 & 12.41 & 9.487 \\
9.0 & 12.82 & 9.135 \\
10.0 & 13.35 & 8.850 \\
12.0 & 14.68 & 8.454 \\
14.0 & 16.23 & 8.209 \\
16.0 & 17.91 & 8.048 \\
18.0 & 19.67 & 7.939 \\
20.0 & 21.49 & 7.863 \\
22.0 & 23.34 & 7.804 \\
24.0 & 25.22 & 7.762 \\
26.0 & 27.12 & 7.727 \\
30.0 & 30.97 & 7.678 \\
35.0 & 35.82 & 7.639 \\
40.0 & 40.72 & 7.618 \\
50.0 & 50.57 & 7.591 \\
100.0 & 100.28 & 7.549 \\
\hline & &
\end{tabular}

used for the $\mathrm{H} \alpha$ widths. Since our results are significantly different, we give in Table 7 new correction factors.

We make the above assumption and further assume that there are expansion and turbulent fields which contribute in quadrature to the total $F W H M$ of observed line profiles. Then the $F W H M$ of $\mathrm{H} \alpha$ and [N II] are, in velocity units,

$F(\mathrm{H} \alpha)^{2}=8 \ln 2\left(k T_{\mathrm{e}} / m_{\mathrm{H}}\right)+\delta^{2}+v_{\mathrm{e}}^{2}+v_{\mathrm{t}}^{2}$,

$F(\mathrm{~N} \mathrm{II})^{2}=8 \ln 2\left(k T_{\mathrm{e}} / m_{\mathrm{N}}\right)+v_{\mathrm{e}}^{2}+v_{\mathrm{t}}^{2}$,

where $\delta$ is defined as that width which adds in quadrature to broaden the $\mathrm{H} \alpha$ line to allow for the fine-structure, $v_{\mathrm{e}}$ and $v_{\mathrm{t}}$ are the $F W H M$ of expansion and turbulent velocity fields, and $m_{\mathrm{H}}$ and $m_{\mathrm{N}}$ are the masses of the hydrogen and nitrogen atoms respectively. From Eqs. (7)-(8), the solution for the electron temperature is $T_{\mathrm{e}}=\frac{\left(F(\mathrm{H} \alpha)^{2}-\delta^{2}-F(\mathrm{~N} \mathrm{II})^{2}\right)}{\left(8 k \ln 2\left[1 / m_{\mathrm{H}}-1 / m_{\mathrm{N}}\right]\right)}$.
The reason that a single value of $\delta$ cannot be given in Table 7 is that the presence of seven unequal components within the line produces a complex effect on the final $F W H M$. We have computed $\delta$ by convolving our computed $\mathrm{H} \alpha$ component intensities with a Gaussian profile corresponding to a total velocity $v_{\text {broad }}$ which represents the combined effect of all broadening processes other than the fine-structure (thermal, instrumental, expansion and turbulent). We determine the $F W H M$ of the resultant profile empirically by searching the profile for the points at half peak intensity. The quantity $\delta$ is then obtained by subtracting $v_{\text {broad }}$ from the $F W H M$ of the profile in quadrature and is thus the effective additional velocity width of the profile due to the fine-structure.

The results are given in Table 7 . It can be seen that $\delta$ is a very slowly-varying function of the total broadening velocity, and so interpolation in the table should be quite accurate.

\section{References}

Baker J.G., Menzel D.H., 1938, ApJ 88, 52

Brink D.M., Satchler G.R., 1968, "Angular Momentum". Oxford University Press

Courtes G., Louise R., Monnet G., 1968, Ann. d'Ap. 31, 493

Cumming R.J., Meikle W.P.S., 1993, MNRAS 262, 689

Dyson J.E., Meaburn J., 1971, A\&A 12, 219

Erickson G.W., 1977, J. Phys. Chem. Ref. Data 6, 831

Ferguson A.I., 1986, Phys. Bull. 37, 330

Gallagher J.S., Hunter D.A., 1983, ApJ 274, 141

Hänsch T.W., Lee S.A., Wallenstein R., Wieman C., 1975, Phys. Rev. Lett. 34, 307

Hummer D.G., Storey P.J., 1987, MNRAS 224, 801

Laming J.M., Feldman U., 1993, ApJ 403, 434

Martin P.G., 1988, ApJS 66, 125

Meaburn J., 1970, Nat 228, 44

Onello J.S., Phillips J.A., 1993, Proc. 104th ASP Meeting, Casssinelli J.P. \& Churchwell E.B. (eds.) ASP Conf. Ser. 35,366

Smits D.P., 1991, MNRAS 248, 193

Storey P.J., Hummer D.G., 1988, MNRAS 231, 1139

Storey P.J., Hummer D.G., 1991, Comp. Phys. Commun. 66, 129

Storey P.J., Hummer D.G., 1995, MNRAS 272, 41

Treffers R.R., 1981, PASP 93, 247

Williams R.E., Woolf N.J., Hege E.K., Moore R.L., Kopriva D.A., 1978, ApJ 224, 171

Wilson O.C., Munch G., Flather E.M., Coffeen M.F., 1959, ApJS 4, 199 